\title{
Thermotropic liquid crystalline polyesters derived from bis-(4-hydroxybenzoyloxy)-2-methyl-1,4-benzene and aliphatic dicarboxylic acid chlorides
}

\author{
KHUDBUDIN MULANI ${ }^{1}$, MOHASIN MOMIN ${ }^{1}$, NITIN GANJAVE ${ }^{2}$ and NAYAKU CHAVAN ${ }^{1}$ ** \\ ${ }^{1}$ Polymer Science and Engineering Division, CSIR-National Chemical Laboratory, Pune 411 008, India \\ ${ }^{2}$ Department of Chemistry, Fergusson College, F.C. Road, Pune 411 004, India
}

MS received 8 February 2015; accepted 15 April 2015

\begin{abstract}
A series of thermotropic liquid crystalline polyesters derived from bis-(4-hydroxybenzoyloxy)-2methyl-1,4-benzene (BHBOMB) and aliphatic dicarboxylic acid chlorides were investigated. All these polyesters were synthesized by interfacial polycondensation method and characterized by differential scanning calorimetry and wide-angle $X$-ray diffractometer. These polyesters consist of BHBOMB as a mesogenic diol and aliphatic diacid chlorides were used as flexible spacers. The length of oligomethylene units in polymer was varied from the trimethylene to the dodecamethylene groups. The transition temperatures and thermodynamic properties were studied for all these polymers. All these polyesters were soluble in chlorinated solvents such as chloroform, dichloromethane, dichloroethane, etc. More importantly, all these polyesters exhibited very large mesophase stability.
\end{abstract}

Keywords. Liquid crystalline polyesters; thermotropic; interfacial polycondensation; rigid diol; dicarboxylic acid chloride.

\section{Introduction}

In the recent past, a branch of polymer chemistry, i.e., synthesis of liquid crystalline polymers (LCPs) has received more attention. LCPs are broadly divided into two main classes, namely thermotropic (TLCPs) and lyotropic (LLCPs) depending on the phenomena of formation of liquid crystalline phase. LLCPs exhibit liquid cystallinity in the solution, whereas TLCPs show liquid crystallinity in the melt. ${ }^{1,2}$ Researchers concentrate on modifing the structure and properties of liquid crystalline aromatic polymers. ${ }^{3-5}$ The thermotropic liquid crystalline behaviour of polymers is of strong interest among polymer scientists, because of their outstanding thermal and chemical resistance, high strength as well as low linear viscosity in liquid crystalline state. ${ }^{6-13}$ Particularly, these high-performance liquid crystalline aromatic polyesters have found wide range of applications in the variety of fields, such as, transportation industry, construction industry, food containers as well as electric and electronic industry and other applications, requiring chemical inertness and high strength. ${ }^{14}$ Due to low relative dielectric constants and low dissipation factors, these types of polyesters are specifically attractive for microwave frequency electronics. ${ }^{15}$ Broadly, TLCPs constitute from two different structural moieties: flexible and

\footnotetext{
*Author for correspondence (nn.chavan@ ncl.res.in)
}

rigid. Flexible aliphatic spacer groups are used to insulate the intramolecular interaction in the liquid crystalline portion, whereas rigid moiety incorporated in main chain as mesogen to exhibit liquid crystalline phase. In particular, flexible spacers are introduced into the polymer backbone to reduce the transition temperature as well as to improve processability.

In TLCPs the substituent effects are of special interest, as crystal-liquid crystal transition temperature of fully extended rigid rod polymers are extremely high. It is desirable to lower the crystal-liquid crystal temperature from the processing point of view. The effect of alkyl substitution on their glass transition temperature, melting temperature, melting points and thermal stabilities is discussed in detail somewhere. ${ }^{16}$ They also studied polymer with alkyl substitution more than eight carbon atoms and observed that longer alkyl substitution does not form a liquid crystalline phase. The effect of alkyl substitution on azoxy-based thermotropic liquid crystallineordered polyesters was examined and observed considerable decrease in the crystal-liquid crystal transition temperature. ${ }^{17}$

Several strategies have been used to decrease the liquid crystalline phase transition temperature. Substitution on mesogenic segment is one of the methodologies used for this purpose. As melting temperature of fully extended rigid rod polymers is very high, thus, majority of such polymers degrade before its isotropization temperature. 
Substitution lowers phase transition temperature substantially due to decrease in aspect ratio of rigid block. It is desirable to lower the melting temperature for processing through conventional methods. This could be accomplished by introducing substituent on the mesogenic unit of the polymer. ${ }^{18}$

The mesogen is referred as rigid moiety in liquid crystalline molecule. It is rod-like structure composed of two or more aromatic rings connected in one direction. Rigid part aligns molecule in one direction, whereas flexible part induces fluidity in the liquid crystal. Transition temperatures of mesomorphic polymers have been enhanced by an increase in the number of aromatic rings in the main chain. ${ }^{19}$ However, the presence of large number of aromatic rings results in non-melting polymers. Crystalliquid crystal, liquid crystal-isotropic transition temperature and mesophase stability of these LCPs are higher. In the solution polycondensation method, mesogen and acid chloride lie in same phase, whereas in the interfacial polycondensation technique, acid chloride introduces in organic phase and diol is added into the aqueous phase and form two immiscible phases, and both phases were mixed together by introducing phase transfer catalyst. Polymerization conditions affect the polymer properties such as molecular weight, polydispersity and crystallinity. ${ }^{20}$

In the present study, we report the synthesis and characterization of thermotropic LCPs prepared from bis-(4hydroxybenzoyloxy)-2-methyl-1,4-benzene (BHBOMB) mesogenic moiety and diacid chloride is used as flexible spacer to dissociate the disorder of the main chain from the greater order of mesogenic group and decouple the motion of mesogenic moiety from polymer backbone. Flexible spacers such as glutaryl, adipoyl, pimeloyl, suberoyl, azeloyl, sebacoyl and decanedioyl chlorides have been used to separate mesogenic group in the main chain from each other. In this article, the thermal transition temperatures, liquid crystallinity and crystallinity of the mesophasic polymers are also described.

\section{Experimental}

\subsection{Materials}

Briefly, 2-methyl hydroquinone and 4-hydroxybenzoic acid were purchased from Aldrich and purified by recrystallization from acetone and hot distilled water, respectively. Acetic anhydride, dichloromethane, methanol, pyridine, chloroform and potassium hydroxide were procured from Merck and used as-received. All acids used for synthesis of acid chlorides were purchased from Aldrich and used without purification.

\subsection{Synthesis of $B H B O M B$}

Trimesogenic diol BHBOMB was synthesized using $p$-hydroxybenzoic acid as starting material and 2-methyl hydroquinone as central moiety. ${ }^{21}$ The synthesis route of BHBOMB is depicted in scheme 1 . The detailed procedure is as follows.

2.2a Synthesis of 4-acetoxybenzoic acid [4-ACBA]: 4Hydroxybenzoic acid, $13.81 \mathrm{~g}(0.1 \mathrm{~mol})$ and $250 \mathrm{ml}$ distilled water were added in $500 \mathrm{ml}$ beaker and stirred to make uniform slurry. Into another beaker, $4.4 \mathrm{~g}$ of sodium hydroxide $(0.11 \mathrm{~mol})$ was dissolved in $50 \mathrm{ml}$ distilled water, added to the slurry, and stirred to dissolve 4hydroxybenzoic acid as to form sodium salt. Distilled acetic anhydride, $11.23 \mathrm{~g}(0.11 \mathrm{~mol})$ was then added and stirred for $4 \mathrm{~h}$ at room temperature to obtain a precipitate of 4-acetoxybenzoic acid. The precipitate was filtered, washed several times with cold dilute hydrochloric acid and distilled water. The crude product was recrystallized from methanol and dried under reduced pressure. Yield: $80 \%$. Melting point: $190^{\circ} \mathrm{C}$ (observed); $190-192^{\circ} \mathrm{C}$ (reported). ${ }^{21}$ IR $\left(\mathrm{KBr}, \mathrm{cm}^{-1}\right): 1730 \quad(\mathrm{C}=\mathrm{O}) .{ }^{1} \mathrm{H} \quad \mathrm{NMR}$ $\left(400 \mathrm{MHz}, \mathrm{DMSO}_{\mathrm{d}}\right.$ ) $: \delta 8.00[2 \mathrm{H}, \mathrm{d}], 7.30[2 \mathrm{H}, \mathrm{d}], 2.30$ [3H, s], $10.10[1 \mathrm{H}, \mathrm{s}]$.

2.2b Synthesis of 4-acetoxybenzoyl chloride: 4Acetoxybenzoic acid, $9.0 \mathrm{~g}(0.05 \mathrm{~mol})$, was charged into a single-necked $100 \mathrm{ml}$ round bottom flask. Thionyl chloride, $5 \mathrm{ml}(0.07 \mathrm{~mol})$, was added drop wise to the reaction flask and reaction mixture refluxed gently for $8 \mathrm{~h}$. The initial heterogeneous mass homogenized to give a yellowish fluid. Excess thionyl chloride was removed by normal distillation. The crude acid chloride was purified by double distillation under reduced pressure to get colourless liquid. Yield: $90 \%$. Melting point: $29-30^{\circ} \mathrm{C}$ (observed); $29-$ $30^{\circ} \mathrm{C}$ (reported). ${ }^{21} \mathrm{IR}\left(\mathrm{KBr}, \mathrm{cm}^{-1}\right): 1780(\mathrm{COCl}), 1730$ $(\mathrm{C}=\mathrm{O})$ and $1370\left(-\mathrm{CH}\right.$, bending). ${ }^{1} \mathrm{H} \mathrm{NMR}(400 \mathrm{MHz}$, $\left.\mathrm{CDCl}_{3}\right): \delta 2.1[3 \mathrm{H}, \mathrm{s}], 7.1[2 \mathrm{H}, \mathrm{d}]$ and $8.0[2 \mathrm{H}, \mathrm{d}]$.

2.2c Synthesis of bis-(4-acetoxybenzoloxy)-2-methyl1,4-benzene: 2-Methyl hydroquinone (4.4 g, $0.04 \mathrm{~mol})$

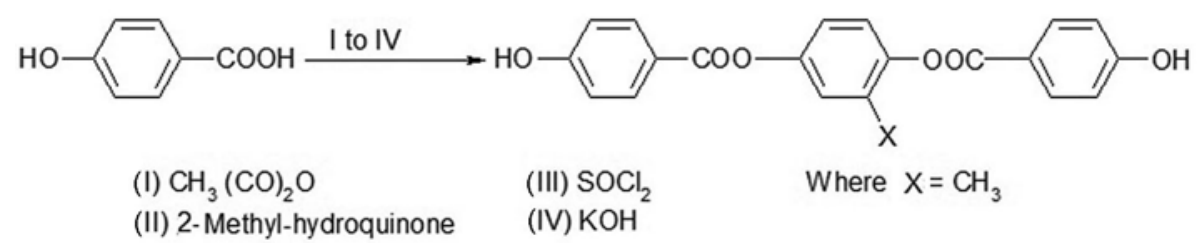

Scheme 1. Synthesis of bis-(4-hydroxybenzoyloxy)-2-methyl-1,4-benzene (BHBOMB) mesogen. 
and $25 \mathrm{ml}$ pyridine $(0.3 \mathrm{~mol})$ were charged into $500 \mathrm{ml}$ three-necked round bottom flask equipped with magnetic stirrer, nitrogen inlet and calcium chloride guard tube. Into another flask, $23.82 \mathrm{~g}$ of 4-acetoxybenzoyl chloride $(0.12 \mathrm{~mol})$ and $300 \mathrm{ml}$ of dry 1,2-dichloroethane were taken. The acid chloride solution was added to the 2methyl-hydroquinone solution. This reaction mixture was stirred under nitrogen blanket for $48 \mathrm{~h}$ at room temperature. The reaction mixture was then washed sequentially with 5\% sodium carbonate solution, 5\% hydrochloric acid and distilled water. 1,2-Dichloroethane layer was evaporated to dryness on rotavapor. Crude product was recrystallized from chloroform/petroleum ether $(60: 80)$ solvent mixture. The product was filtered and dried in vacuum oven at $80^{\circ} \mathrm{C}$ for $4 \mathrm{~h}$. Yield: $90 \%$. Melting point: $108^{\circ} \mathrm{C}$ (observed); ${ }^{6} \mathrm{IR}\left(\mathrm{KBr}, \mathrm{cm}^{-1}\right): 1720(\mathrm{C}=\mathrm{O}$ stretching) and $820\left(\mathrm{C}-\mathrm{CH}_{3}\right) .{ }^{1} \mathrm{H}$ NMR $\left(400 \mathrm{MHz}, \mathrm{CDCl}_{3}\right): \delta$ $2.2(3 \mathrm{H}, \mathrm{s}), 2.3(6 \mathrm{H}, \mathrm{s}), 7.4(8 \mathrm{H}, \mathrm{d})$ and $8.4(3 \mathrm{H}, \mathrm{d})$.

2.2d Synthesis of BHBOMB: Potassium hydroxide $(1.32 \mathrm{~g}, 0.02 \mathrm{~mol})$ and $100 \mathrm{ml}$ methanol were charged into $250 \mathrm{ml}$ single-necked round bottom flask equipped with magnetic stirrer and kept in ice bath and stirred till it dissolved completely. Alcoholic potassium hydroxide solution was cooled to $0-5^{\circ} \mathrm{C}$. Into reaction flask, $4.34 \mathrm{~g}(0.01 \mathrm{~mol})$ of bis-(4-acetoxybenzoyloxy)-2-methyl-1,4-benzene was added and stirred at $0-5^{\circ} \mathrm{C}$ for $4 \mathrm{~h}$. Reaction mixture was acidified with $4 \mathrm{~N}$ hydrochloric acid. Methanol was removed under reduced pressure. The crude product was dissolved in $400 \mathrm{ml}$ ethyl acetate and solution washed three times with distilled water. Ethyl acetate was distilled off and the solid was extracted with chloroform. Distilled off the chloroform to get purified product. The solid crude product was washed with methanol and dried in vacuum oven at $80^{\circ} \mathrm{C}$ for $4 \mathrm{~h}$. Yield: $75 \%$. Melting point: $280 \pm 1{ }^{\circ} \mathrm{C}$ (observed); $280^{\circ} \mathrm{C}$ (reported). ${ }^{6} \mathrm{IR}(\mathrm{KBr}$, $\left.\mathrm{cm}^{-1}\right): 3390$ (-OH stretching), $1700(\mathrm{C}=\mathrm{O})$ and 820
$\left(\mathrm{C}-\mathrm{CH}_{3}\right) .{ }^{1} \mathrm{H}$ NMR $\left(400 \mathrm{MHz}, \mathrm{DMSO}_{6}\right): \delta 2.2(3 \mathrm{H}, \mathrm{s})$, $6.9(4 \mathrm{H}, \mathrm{d}), 7.2(3 \mathrm{H}, \mathrm{s})$ and $8.0(4 \mathrm{H}, \mathrm{dd})$ and $10.6(2 \mathrm{H}, \mathrm{s})$.

2.2e Preparation of aliphatic diacid chlorides: Glutaroyl chloride, adipoyl chloride, pimeloyl chloride, suberoyl chloride, azeloyl chloride, sebacoyl chloride and dodecanedioyl chloride were synthesized from the parent diacids using thionyl chloride. The aliphatic diacids were gently refluxed with thionyl chloride at $80^{\circ} \mathrm{C}$ for $4 \mathrm{~h}$ to get clear solution. This acid chloride solution was initially distilled with lower reduced pressure $(100-150 \mathrm{mmHg}$ ) to remove the excess of thionyl chloride and was double distilled under reduced pressure $(1-2 \mathrm{mmHg})$ at $150^{\circ} \mathrm{C}$ till get colourless product, prior to use.

\subsection{Interfacial polycondensation}

The combined type polyester was synthesized according to scheme 2. Mole ratios of rigid diol BHBOMB and different aliphatic diacid chlorides for interfacial polycondensation are represented in table 1. BHBOMB (1.4573 g, $0.04 \mathrm{~mol})$, sodium hydroxide $(0.008 \mathrm{~mol})$ and benzyl triethyl ammonium chloride (BTEAC) (0.02 g) were charged into two-necked $100 \mathrm{ml}$ round bottom flask equipped with mechanical stirrer, nitrogen inlet, and calcium chloride guard tube, and stirred till it dissolved completely in $15 \mathrm{ml}$ aqueous phase. Into another stoperred flask, organic layer consisting of $0.04 \mathrm{~mol}$ of acid chloride in $15 \mathrm{ml}$ dry 1,2-dichloroethane (DCE) was added. The reaction mixture was vigorously stirred using 6000-7000 rpm for $30 \mathrm{~min}$ at $0-5^{\circ} \mathrm{C}$. The polyester was precipitated by pouring into $500 \mathrm{ml}$ methanol. It was filtered, washed with $5 \mathrm{wt} \%$ sodium carbonate solution, dilute hydrochloric acid and distilled water. The soxhlet extraction was carried out with methanol and followed by drying in vacuum oven at $80^{\circ} \mathrm{C}$ for $8 \mathrm{~h}$.
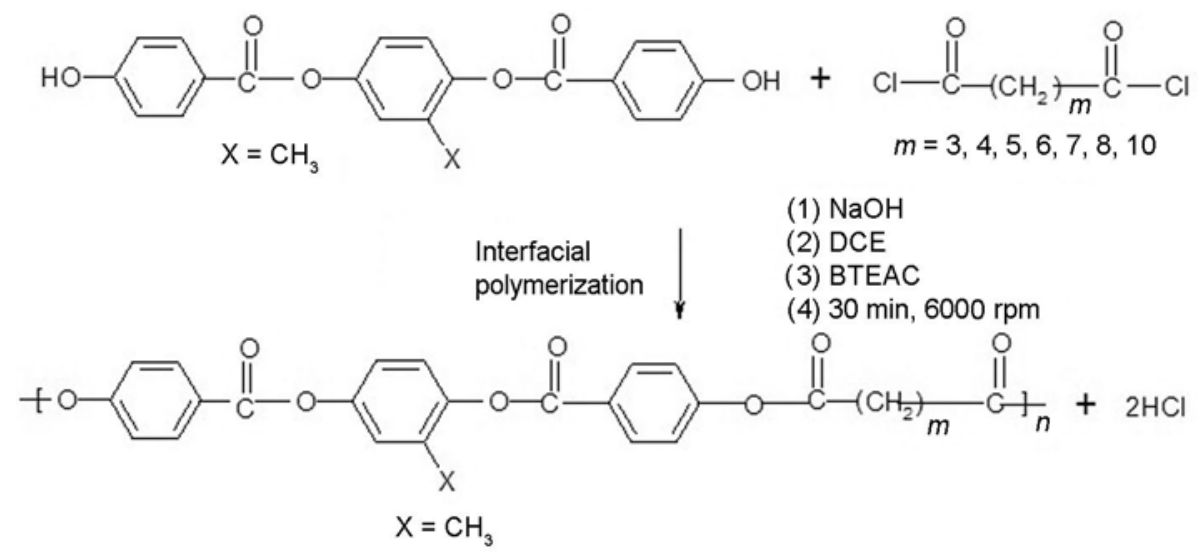

Scheme 2. Synthesis pathway of polyesters using BHBOMB and aliphatic diacid chlorides. 
Table 1. Mole ratio of BHBOMB and aliphatic diacid chlorides for interfacial polycondensation.

\begin{tabular}{lcclcccc}
\hline \multirow{2}{*}{$\begin{array}{l}\text { Polymer } \\
\text { code }\end{array}$} & \multicolumn{2}{c}{ BHBOMB } & & \multicolumn{2}{c}{ Aliphatic diacid chloride } & \\
\cline { 2 - 3 } & Mole & $\mathrm{g}$ & & Name & Mole & $\mathrm{g}$ & $\begin{array}{c}\text { Polyester } \\
\left(\mathrm{m} \mathrm{ru}^{-1}\right)^{*}\end{array}$ \\
\hline PE-101 & 0.04 & 1.4573 & & Glutaryl & 0.4 & 0.6760 & 460.41 \\
PE-102 & 0.04 & 1.4573 & & Adipoyl & 0.4 & 0.7321 & 474.44 \\
PE-103 & 0.04 & 1.4573 & & Pimeloyl & 0.4 & 0.7882 & 488.46 \\
PE-104 & 0.04 & 1.4573 & & Suberoyl & 0.4 & 0.8443 & 502.49 \\
PE-105 & 0.04 & 1.4573 & & Azeloyl & 0.4 & 0.9004 & 516.52 \\
PE-106 & 0.04 & 1.4573 & & Sebacoyl & 0.4 & 0.9565 & 530.54 \\
PE-107 & 0.04 & 1.4573 & & Dodecane & 0.4 & 1.0687 & 558.59 \\
\hline
\end{tabular}

*Mole per repeat unit.

Table 2. Transition and thermodynamic data of BHBOMB polyester synthesized by interfacial polycondensation (first heating cycle).

\begin{tabular}{lccccccccc}
\hline Polymer code & $\mathrm{K}-\mathrm{N}\left({ }^{\circ} \mathrm{C}\right)$ & $\mathrm{N}-\mathrm{I}\left({ }^{\circ} \mathrm{C}\right)$ & $\Delta T\left({ }^{\circ} \mathrm{C}\right)$ & $\Delta H, \mathrm{~K}-\mathrm{N}$ & $\Delta H, \mathrm{~N}-\mathrm{I}$ & $\mathrm{K}-\mathrm{N}$ & $\Delta S, \mathrm{~N}-\mathrm{I}$ & {$\left[\mathrm{CH}_{2}\right]_{n}$} & $\mathrm{DOC} \%$ \\
\hline PE-101 & 208.3 & 355.2 & 146.9 & 9.58 & 0.28 & 19.20 & 0.44 & 3 & 54.60 \\
$\mathrm{PE}-102$ & 186.4 & 349.5 & 163.1 & 3.75 & - & 8.15 & - & 4 & 42.93 \\
PE-103 & 173.3 & 327.5 & 154.2 & 6.55 & 1.54 & 14.67 & 2.57 & 5 & 37.15 \\
$\mathrm{PE}-104$ & 199.1 & 325.7 & 126.6 & 4.07 & 4.88 & 8.62 & 8.14 & 6 & 44.44 \\
$\mathrm{PE}-105$ & 164.3 & 289.9 & 125.6 & 12.35 & 5.17 & 28.23 & 9.18 & 7 & 40.60 \\
PE-106 & 182.8 & 291.0 & 108.2 & 3.02 & 7.69 & 6.63 & 13.64 & 8 & 38.60 \\
PE-107 & $184.6^{\mathrm{a}}$ & $274.4^{\mathrm{b}}$ & 89.8 & $5.92^{\mathrm{a}}$ & $6.93^{\mathrm{b}}$ & $12.94^{\mathrm{a}}$ & $12.65^{\mathrm{b}}$ & 10 & 46.15 \\
\hline
\end{tabular}

$\mathrm{K}-\mathrm{N}\left({ }^{\circ} \mathrm{C}\right)$ : Crystal-liquid crystal transition temperature, $\mathrm{N}-\mathrm{I}\left({ }^{\circ} \mathrm{C}\right)$ : Liquid crystal-isotropic transition temperature, $\Delta T\left({ }^{\circ} \mathrm{C}\right)$ : Temperature range of metaphase stability, $\Delta H(\mathrm{~K}-\mathrm{N})$ and $\Delta H(\mathrm{~N}-\mathrm{I})$ : Enthalpy change in $\mathrm{kJ} \mathrm{mol}^{-1}$ of repeat unit $(\mathrm{mru}), \Delta S(\mathrm{~K}-\mathrm{N})$ and $\Delta S$ $(\mathrm{N}-\mathrm{I})$ : Entropy change in $\mathrm{J}_{\mathrm{mru}^{-1}}(\mathrm{~K})$, DOC: Percent degree of crystallinity.

${ }^{\mathrm{a}}$ Crystal to smectic transition and ${ }^{\mathrm{b}} \mathrm{Smectic}$ to isotropic transition.

\subsection{Characterization}

Synthesized polyesters were soluble in typical organic solvents such as chloroform, carbon tetrachloride, dichloromethane, dichloroethane, 4-chlorophenol or phenol : tetrachloroethane $(50: 50 \mathrm{v} / \mathrm{v})$ mixture. The thermal behaviour was determined with a Mettler DSC-30 thermal analyser under nitrogen atmosphere. Indium and zinc standards were used to calibrate the DSC temperature and enthalpy scale. The endotherm peak maxima were selected as phase transition temperatures. The enthalpies and entropies of liquid crystalline phase transitions were determined. A Leitz Ortholux polarizing microscope with a Mettler FP-52 hot stage controlled by Mettler FP-5 temperature controller was used for visual examination of phase changes in the polymers. Wide-angle X-ray diffractometer was used to determine the degree of crystallinity at room temperature. Inherent viscosities were determined with $0.5 \%$ solution in chloroform at $30^{\circ} \mathrm{C}$.

\section{Results and discussion}

\subsection{General properties}

The transition temperatures and thermodynamic properties are presented in tables 2 and 3. These polymers were soluble in all chlorinated solvents, namely chloroform, dichloromethane, dichloroethane, 4-chlorophenol, phenol: tetrachloroethane $(50: 50, \mathrm{v} / \mathrm{v})$ solvent mixture. Main chain thermotropic liquid crystalline polyesters exhibit complex and sometimes uninterpretable thermograms for crystal-liquid crystal transitions. ${ }^{17,22}$ The factors responsible for such complex thermal behaviour are solid-solid transitions, melting of crystals/recrystallization of new ones, formation in rapid succession and more than one liquid crystalline phase. All polyesters exhibited a rather narrow endothermic transition for the mesophase at the isotropization temperature, which suggests that polydispersity were rather narrow.

DSC thermograms of first heating cycle of BHBOMB homopolyesters are presented in figure 1, whereas DSC thermograms of second heating cycle of BHBOMB homopolyesters are depicted in figure 2 . The melting endotherms were pleasingly simple (unstructured). In general, the nature of thermograms of first and second heating cycles is almost similar, when compared, respectively. Transition temperatures of second heating cycles are slightly higher than first heating cycle. This is due to annealing of crystals in crystal to liquid crystal transition as well as liquid crystal to isotropic. Mesophase stability was also higher in second heating cycle compare to first heating cycle. The polyesters synthesized are well 
Table 3. Transition and thermodynamic data of BHBOMB polyester synthesized by interfacial polycondensation (second heating cycle).

\begin{tabular}{lcccccrccc}
\hline Polymer code & $\mathrm{K}-\mathrm{N}\left({ }^{\circ} \mathrm{C}\right)$ & $\mathrm{N}-\mathrm{I}\left({ }^{\circ} \mathrm{C}\right)$ & $\Delta T\left({ }^{\circ} \mathrm{C}\right)$ & $\Delta H, \mathrm{~K}-\mathrm{N}$ & $\Delta H, \mathrm{~N}-\mathrm{I}$ & $\Delta S, \mathrm{~K}-\mathrm{N}$ & $\Delta S, \mathrm{~N}-\mathrm{I}$ & {$\left[\mathrm{CH}_{2}\right]_{n}$} & $\mathrm{IV}$ \\
\hline PE-101 & 210.6 & 350.3 & 139.7 & 4.42 & 1.24 & 11.43 & 1.99 & 3 & 0.97 \\
PE-102 & 186.3 & 369.5 & 183.2 & 3.84 & - & 8.37 & - & 4 & 0.93 \\
PE-103 & 173.3 & 351.4 & 178.1 & 3.81 & 1.66 & 8.54 & 2.66 & 5 & 1.46 \\
PE-104 & 195.5 & 349.5 & 154.0 & 2.71 & 7.24 & 5.79 & 11.62 & 6 & 1.10 \\
PE-105 & 164.1 & 311.0 & 146.9 & 8.73 & 5.68 & 19.97 & 8.70 & 7 & 1.03 \\
PE-106 & 177.2 & 312.9 & 135.7 & 2.86 & 6.74 & 6.36 & 11.50 & 8 & 0.98 \\
PE-107 & $188.2^{\mathrm{a}}$ & $294.6^{\mathrm{b}}$ & 106.4 & $6.31^{\mathrm{a}}$ & $8.94^{\mathrm{b}}$ & $8.24^{\mathrm{a}}$ & $15.75^{\mathrm{b}}$ & 10 & 1.05 \\
\hline
\end{tabular}

$\mathrm{K}-\mathrm{N}\left({ }^{\circ} \mathrm{C}\right)$ : Crystal-liquid crystal transition temperature, $\mathrm{N}-\mathrm{I}\left({ }^{\circ} \mathrm{C}\right)$ : Liquid crystal-isotropic transition temperature, $\Delta T\left({ }^{\circ} \mathrm{C}\right)$ : Temperature range of metaphase stability, $\Delta H(\mathrm{~K}-\mathrm{N})$ and $\Delta H(\mathrm{~N}-\mathrm{I})$ : Enthalpy change in $\mathrm{kJ} \mathrm{mol}^{-1}$ of repeat unit $(\mathrm{mru}), \Delta S(\mathrm{~K}-\mathrm{N})$ and $\Delta S$ $(\mathrm{N}-\mathrm{I})$ : Entropy change in $\mathrm{J} \mathrm{mru}^{-1}(\mathrm{~K})$, IV: Intrinsic viscosity (at $30^{\circ} \mathrm{C}$ in $\mathrm{CHCl}_{3}$ ).

${ }^{\mathrm{a}}$ Crystal to smectic transition and ${ }^{\mathrm{b}} \mathrm{Smectic}$ to isotropic transition.

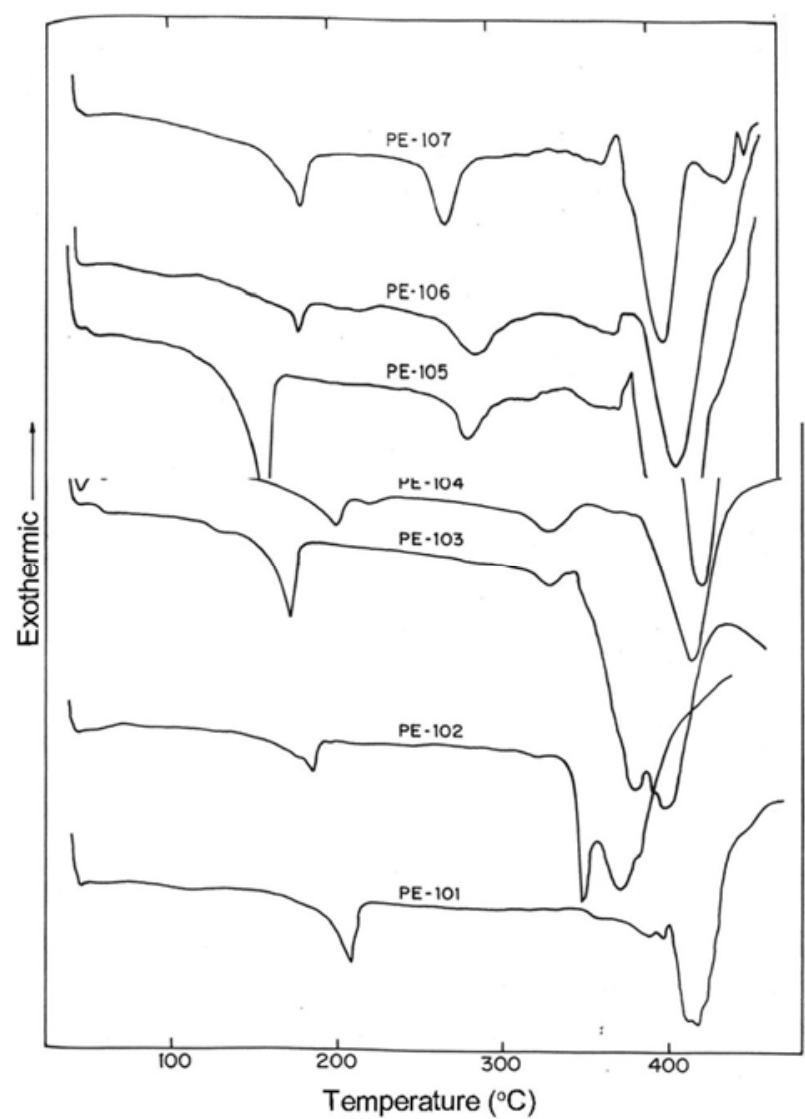

Figure 1. DSC thermograms of first heating cycle of BHBOMB homopolyesters.

chemically ordered. A broadening of isotropic transition would be expected for polydisperse samples, as indeed observed for the polyesters based on unsubstituted trimesogen, wherein insolubility intervened. The peak maxima was selected to make comparative estimates of structureproperty relationship. We were unable to study the transition from isotropic to the liquid crystal state (deisotropization process) as the isotropizations in most samples in this series are rapidly followed by decomposition. It is

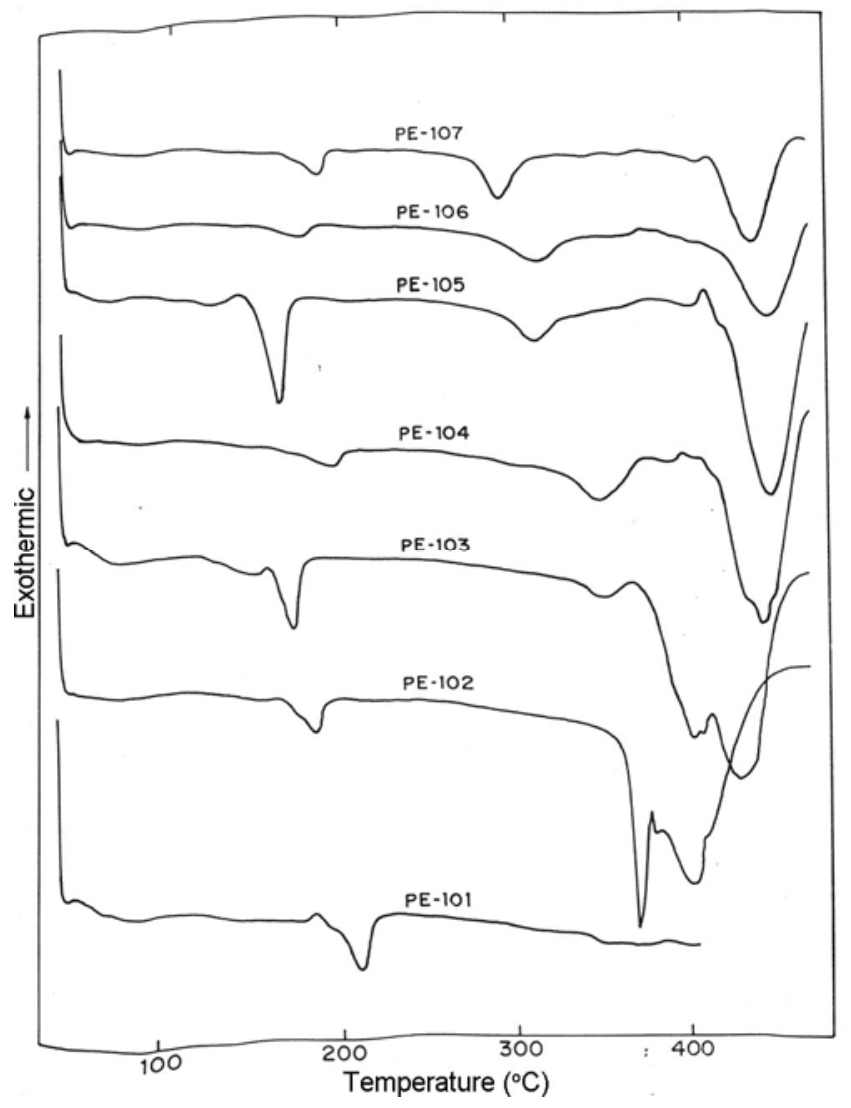

Figure 2. DSC thermograms of second heating cycle of BHBOMB homopolyesters.

known that deisotraopization in main liquid crystalline polyesters exhibit a smaller degree of super cooling. In other words, isotropization is thermodynamically more of an equilibrium (reversible) process, rather than crystal to liquid crystal transition. The crystallization in these polyesters, when cooled at the rate of $10^{\circ} \mathrm{C} \mathrm{min}{ }^{-1}$ from $250^{\circ} \mathrm{C}$, showed moderate supercooling of about $50^{\circ} \mathrm{C}$.

All polyesters formed a turbid melt showing a strong stiropalescence up to the isotropization or clearing temperature $\left(T_{\mathrm{i}}\right)$. Thin films of polymer melts were examined on 
the hot stage of a polarizing microscope for optical textures. Polyesters other than PE-107 showed characteristic features pertaining to the nematic mesophase, i.e., threaded-schlieren texture. Polyesters with longer $(n=10)$ flexible spacers, such as PE-107, showed focal conic texture typical of smectic mesophase prior to the isotropic transition and is depicted in figure 3. Photomicrograph of PE-107 was observed at $152.4^{\circ} \mathrm{C}$ during cooling cycle. Thus, the longest flexible spacer in this series $(n=10)$ allowed the rigid mesogenic unit to align more readily in two-dimensional layers giving rise to a smectic mesophase.

\subsection{Substitution effect}

The liquid crystalline behaviour of low-molecular mass liquid crystals with different substituents on mesogenic units has been systematically studied by $\mathrm{Gray}^{23,24}$ and others. $^{25-27}$ In general, the influence of substitution is complicated by both steric and polar effects. The steric effect, which leads to a less thermally stable mesophase, may involve in following contribution: (i) a broadening of the mesogenic groups by the substituent and therefore, a decrease in the overall length to diameter ratio; (ii) a decrease in the coplanarity of adjacent mesogenic moieties due to steric interaction between substituents and (iii) a tendency for the substituent to force apart mesogenic groups in neighbouring polymer chains due to spatial requirements. On the other hand, substituent that imparts an increased polarizability and stronger dipolar interaction between the mesogenic groups acquire stronger intermolecular attractions. This leads to higher thermal stabilities for both crystalline and liquid crystalline phases and results in higher crystal-liquid crystal and isotropization temperatures.

In TLCPs, the substituent plays an important role to reduce the transition temperature, as crystal-liquid

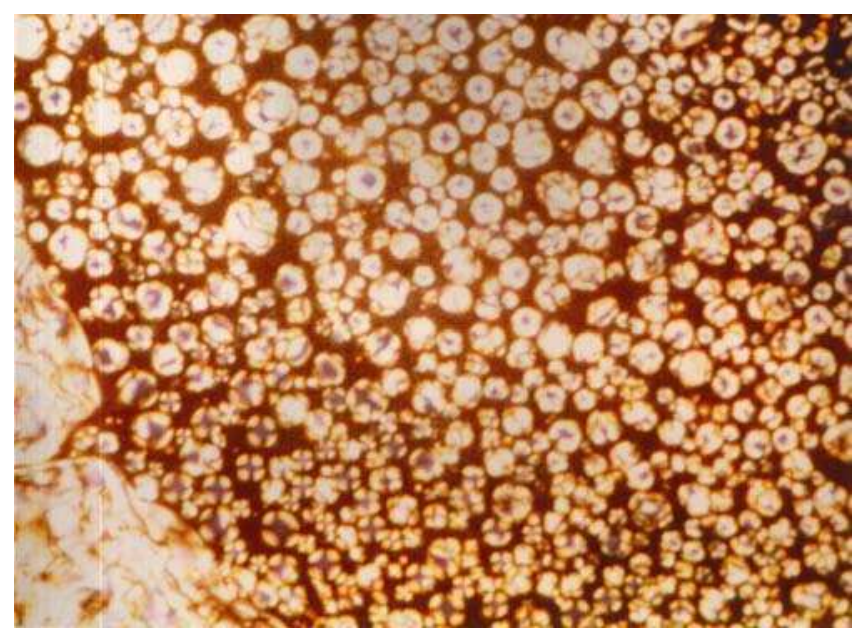

Figure 3. Texture of polyester PE-107 at $152.4^{\circ} \mathrm{C}$. crystal transition temperature of fully extended rigid rod polymers are extremely high. It is desirable to lower the crystal-liquid crystal temperature for processing from liquid crystalline melts. It is apparent that this could be accomplished by introducing substituent into the mesogenic units of the polymer. ${ }^{26}$ The use of monosubstituted hydroquinone in random copolyesters results in a considerable decrease in the crystal-liquid crystal transition temperature and permit melt spinning from the liquid crystalline states. ${ }^{28}$

\subsection{Thermodynamics of LC states}

The transition temperatures, thermodynamic properties as determined by DSC and first heating cycle thermodynamic properties are presented in table 2 . In TLCPs, the crystal and isotropic transition temperatures are dependent on molecular weight till a critical value is attained. The polyesters of this series were soluble and hence it is believed that these had attained sufficiently high molecular weights to enable a meaningful comparison of the thermal and thermodynamic properties.

In these series, all polyesters display moderate, though not very precise, odd-even fluctuation in the crystalliquid crystal transition temperatures $T_{\mathrm{m}}$, relative to methylene unit length except first and last polyesters (PE101 and PE-107). This odd-even effect seems to suggest that the spacer part of the polymer backbone is largely in its most extended (trans) conformation. In this conformation, even numbered methylene spacers can retain their co-linearity with mesogenic parts, while the odd numbered methylene spacers cannot retain their co-linearity with mesogenic parts. It must be mentioned that the oddeven effect in $T_{\mathrm{m}}$ is not universal in homologous LCPs. The off-equilibrium nature of the crystal to liquid crystal transition can mark this odd-even effect to some extent. The isotropization temperature $\left(T_{\mathrm{i}}\right)$ showed more tendency towards a continued decrease rather than an odd-even fluctuation. Unlike liquid, crystal transition of isotropization is a near-equilibrium process. But very often, isotropization reaction occurs at high temperatures with polyesters and risks of concurrent or imminent degradation are not entirely avoidable. The problem of degradation can impair the actual effect in $T_{\mathrm{i}}$. In the present series, the isotropization temperature decreases continuously with the spacer length.

\subsection{Mesophase stability $(\Delta T)$}

Mesophase stability $\left(\Delta T=T_{\mathrm{i}}-T_{\mathrm{m}}\right)$ of polyesters decreases with the increase in methylene units in the flexible spacer. It is due to the dilution of mesogen, indirect reduction in rigidity of polymeric structure. Odd-even effect was also observed in almost all polymers. Even number of methylene units showed higher mesophase stability than 
odd number of methylene units. It is due to even number of methylene units allowed trans-trans conformation, whereas odd number of methylene dictates gauche conformation. The mesophase stability of these polyesters were found to be quite high $\left(>100^{\circ} \mathrm{C}\right)$.

\subsection{Enthalpy and entropy change}

The enthalpy and entropy changes in the first and second heating cycle of polyesters are represented in tables 2 and 3 , respectively. These data do not show any clear and regular trend for crystal to liquid crystal $(\mathrm{K}-\mathrm{LC})$ or for liquid crystal to isotropic (LC-I) transition in either first or second heating cycle. The enthalpy or entropy data for $n=3-10$ indicate a variable degree of supercooling of the nematic to crystal $(\mathrm{N}-\mathrm{K})$ transition, i.e., a variable degree of crystallinity in the supercooled sample prior to the second heating cycle. In other words, our attempts to maintain a constant thermal history in all these samples had not been successful.

The enthalpy and entropy change for the liquid crystal to isotropic transition (LC-I) are more or less comparable in both first and second heating cycles. The values of $\Delta H_{\mathrm{K}-\mathrm{LC}}$ and $\Delta S_{\mathrm{K}-\mathrm{LC}}$ observed in both cycles for all above polyesters discussed are comparable with those found normally in main chain liquid crystalline polyesters. The degradation in polyesters PE-104 to PE-107 generates somewhat elevated values of $\Delta H_{\mathrm{LC}-\mathrm{I}}$ and $\Delta S_{\mathrm{LC}-\mathrm{I}}$. Such elevated values of $\Delta H_{\mathrm{LC}-\mathrm{I}}$ and $\Delta S_{\mathrm{LC}-\mathrm{I}}$ are usually typical of transition from highly ordered smectic phase to the isotropic phase. We observed that the presence of smectic phase in PE-107 in which number of methylene units are 10 , and hence the high values of $\Delta H_{\mathrm{LC}-\mathrm{I}}$ and $\Delta S_{\mathrm{LC}-\mathrm{I}}$ are expected in this case, provided the isotropic transition has occurred from the smectic phase. In other polyesters PE104 to PE-106, textures corresponding only to the nematic phases were observable under hot stage coupled polarizing microscopy. The highest values of $\Delta H_{\mathrm{K}-\mathrm{LC}}$ and $\Delta S_{\mathrm{K}-\mathrm{LC}}$ point to some conformational ordering in the flexible spacer.

Our conclusion is reinforced when we find that $\Delta H_{\mathrm{LC}-\mathrm{I}}$ and $\Delta S_{\mathrm{LC}-\mathrm{I}}$ increase monotonously for the odd and even series of nematic polyesters. The increase for $\Delta H_{\mathrm{LC}-\mathrm{I}}$ is from 0.27 for $n=3 \mathbf{n}$ to $7.69 \mathrm{~kJ} \mathrm{mru}^{-1}$ for $n=8$. Similarly, the increase for $\Delta S_{\mathrm{LC}-\mathrm{I}}$ is from 0.44 to $13.64 \mathrm{~kJ} \mathrm{mru}^{-1}$. The values coated are from the data of the first heating cycle. The increases of odd and even series do not precede with any uniform, average increment of $\Delta H_{\mathrm{LC}-\mathrm{I}}$ and $\Delta S_{\mathrm{LC}-\mathrm{I}}$ per methylene unit. However, the fact remains that there is significant, but not non-uniform, extension of the flexible spacer in the nematic phase of polyesters PE-104 to PE-106. The population of trans conformers in nematic phase could not be estimated due to lack of knowledge about the respective population of trans/gauche conformers in the pretransition state.

\subsection{X-ray diffraction}

The degree of crystallinity data from X-ray diffraction are not always concordance with the values of $\Delta H_{\mathrm{K}-\mathrm{LC}}$ from DSC data. Braggs spacing's less than $5 \AA$ that are seen in all polyesters arise mainly from proffered separation in the lateral packing of the polyesters chains. Spacing of $12 \AA$ or higher indicate regularity of packing along the chain. Lateral packing can allow a regular arrangement of molecules along the chain in spite of the randomness arising out of the positional isomerization of the monosubstituent on the central unit of the trimesogen. The degree of crystallinity in one case overlooks any such order along the chain, because we have not been able to harness any reflection below $\Delta=5^{\circ}$ due to instrumental limitations. It is likely that the crystallinity arising out of this order could have contributed to higher values of $\Delta H_{\mathrm{K}-\mathrm{LC}}$ for PE-103 and PE-105 with $n=5$ and 7, respectively.

\section{Conclusion}

A series of new combined type liquid crystalline polyesters consisting of BHBOMB mesogen by varying length of flexible spacers have been successfully synthesized and characterized. These polyesters exhibit very large mesophasic stability $\left(>100^{\circ} \mathrm{C}\right)$. The experimental data show that endotherms are rather sharp, indicating narrow molecular weight distribution. The polyesters display moderate odd-even fluctuation in liquid crystal transition temperature with the increase in flexible spacer length, while no such effect is observed for the isotropization temperatures. The mesophase stability $\left(\Delta T=T_{\mathrm{i}}-T_{\mathrm{m}}\right)$ decreases regularly with the increase in methylene units. Also, degree of crystallinity decreases with the increase in molecular weights. However, no clear trends were observed for enthalpy and entropy changes with respect to spacer length for either liquid crystal or liquid crystal to isotropic transition due to inability to maintain constant thermal history in all samples. Interestingly, these values are depressed by unsymmetrical monomethyl substitution on the mesogenic core and points to greater disorder induced by the substitution.

\section{References}

1. Gray G W and Winsor P A 1974 Liquid crystals and plastic crystals (New York, USA: John Wiley \& Sons)

2. Gray G W 1987 Thermotropic liquid crystals (New York, USA: John Wiley \& Sons)

3. Chavan N N and Mulani K B 2012 Thermotropic liquid crystalline polysters (Germany: LAP, Lambert Academic Publishing Co., Verlag), ISBN: 978-3-659-28760-2

4. Ciferri A 1961 Liquid crystallinity in polymers (New York, Cambridge: VCH Verlagsgesellschaft Weinheim) 
5. Lenz R W 1985 Polymer J. 17105

6. Chavan N N 1992 PhD thesis (Kolhapur, India: Shivaji University)

7. Zhu Z G, Zhi J G, Liu A H, Cui J X, Tang H, Qiao W Q, Wan X H and Zhou Q F 2007 J. Polym. Sci.: Polym. Chem. 45830

8. Talukdar M 2011 Int. J. Res. Rev. Appl. Sci. 822

9. Thomas E L and Wood B A 1985 Faraday Discuss. Chem. Soc. 79229

10. Strzelecki et D L and Van Luyen 1980 Eur. Polym. J. 16299

11. Roviello A and Sirigu A 1975 J. Polym. Sci.: Lett. Ed. 13455

12. Abet A 1984 Macromolecules 172280

13. Ignatious F, Ward W J, Lenz R W and Kantor S W 1995 Polymer 362819

14. Economy J, Novak B E and Cottis S G 1970 Sampe J. 66

15. Dane C, George E and John P 2004 IEEE Trans. Microwave Theory Tech. 521343

16. Qi-Feng Z and Lenz R W 1983 J. Polym. Sci.: Polym. Chem. 213313

17. Blumstein A, Vilasagar S, Ponrathnam S, Clough S B and Blumstein R B 1982 J. Polym. Sci.: Polym. Phys. Ed. 20 877
18. Yun Y, Jin J and Lee M 1997 J. Polym. Sci.: Polym. Chem. Ed. 352777

19. Krigbaum W R, Kotek R, Ishikawa T and Hakemi H 1984 Eur. Polym. J. 20225

20. Morgan P 1965 Condensation polymers by interfacial and solution polycondensation method (New York: Interscience)

21. Mulani K B, Ganjave N V and Chavan N N 2014 Ind. J. Chem. B53 591

22. Terence T B, Mary E N, Mitchell T and Chun-Che T 1991 J. Phys. Chem. Ref. Data 20189

23. Gray G W and Warrall B M 1959 J. Chem. Soc. 1545

24. Gray G W, Johns B and Marson F 1957 J. Chem. Soc. 393

25. Branch S J, Byron D J, Gray G W, Ibbotson A and Warrall B M 1964 J. Chem. Soc. 3279

26. Arora S L, Ferguson J L and Taylor T R 1970 J. Chem. Soc. 354055

27. Young W R, Haller I and Green D C 1972 J. Chem. Soc. 37 3707

28. Jo B W, Lenz R W and Jin J I 1982 Macromol. Chem. Rapid. Commun. 323 